\title{
IVERMECTIN DISTRIBUTION AND THE CULTURAL CONTEXT OF FOREST ONCHOCERCIASIS IN SOUTH PROVINCE, CAMEROON
}

\author{
BARRY S. HEWLETT, BASILE KOLLO, AND BARNETT L. CLINE \\ Department of Anthropology, Washington State University, Pullman, Washington; Cameroon Ministry \\ of Health, Doula, Cameroon; Center for International Community-Based Studies, Department of \\ Tropical Medicine, Tulane University School of Public Health and Tropical Medicine, New \\ Orleans, Louisiana
}

\begin{abstract}
This investigation examined the cultural context of forest onchocerciasis in several communities in the Dja-Lobo Division of southern Cameroon. The study sought to elucidate behaviors that would enhance or diminish health status relative to forest onchocerciasis and other filarial infections, and to make culturally sensitive and appropriate recommendations regarding the development of health education materials and the long-term sustainability of the ivermectin distribution program in Dja-Lobo. The study consisted of two sequential components; the first was a qualitative study of a few severely affected villages and the second was a quantitative study of 212 randomly selected heads of households from eight villages. The Boulou and Baka peoples in these communities defined general filariasis (minak) as small worms under the skin, identified flies as important transmitters of the illness, and indicated that blindness and other skin and ocular problems were a consequence of the illness. Illness of the Dja (referring to an illness found near the Dja River) was another illness that was closely linked to onchocerciasis; local people indicated it was transmitted by the black flies found near the Dja River, resulting in severe itching and leopard skin. These and other cultural-behavioral data on filariasis were used to implement a health education and distribution program.
\end{abstract}

Successful disease control programs require a high level of understanding of cultural factors that may either enhance or diminish the ability to achieve desired outcomes. Onchocerciasis control efforts currently focus on the development of ivermectin distribution programs, as Merck \& Co., Inc. (Rahway, NJ) is currently providing the drug free-of charge to approved distribution systems. Little is known in Cameroon or elsewhere about the way in which peoples in endemic communities perceive onchocerciasis and its treatment. Especially lacking is information about perceptions of forest onchocerciasis in Africa, which, in contrast to better-known savanna onchocerciasis, is relatively mild, causing less severe disease manifestations. In forest and savanna populations with a similar prevalence, intensity and disease burden are generally lower with forest forms; itching is less severe and skin problems are fewer. Forest populations are also exposed to other filarial diseases, especially loiasis, in addition to onchocerciasis. Their clinical manifestations may be confused with onchocerciasis, and ivermectin may affect these parasites differently.

Sustainability is a challenge for all ivermectin distribution programs because ivermectin does not kill the adult stages of Onchocerca volvulus; to be effective it must be administered on an annual basis to high risk communities over a period of at least 10 years. Sustainability is particularly important with forest onchocerciasis because people must be motivated to seek treatment annually for a relatively mild disease and a minimal threat of blindness.

An additional challenge to the sustainability of ivermectin distribution in Cameroon is the issue of cost-recovery. In response to the Bamako Initiative (devloped by African Ministers of Health to establish cost-effective systems for health care to decrease dependence upon sporadic U.S. and European assistance), the Government of Cameroon has instituted a nationwide, decentralized system of primary health care built upon the essential elements of community co-financing and co-management. Community members participate in the decisions and management of primary health care centers and also are partly responsible for paying for primary health care diagnosis and treatment. While the ivermectin itself is free, the costs of diagnosis, record-keeping, and transportation of the drug to rural areas are considerable. Consequently, a minimal charge for treatment is essential to sustain the program. This potential constraint to the ivermectin distribution program requires an understanding of whether onchocerciasis is perceived as serious enough to pay for an annual preventative treatment, often for the whole family.

Mass distribution of ivermectin was planned for villages that were identified as hyperendemic or mesoendemic ( $>$ $40 \%$ prevalence) by rapid assessment techniques, ${ }^{1}$ while health center-based (passive) distribution was planned for hypoendemic villages. Sustainability was a concern for both distribution systems, in part, because people would be required to pay a minimal fee to participate.

The primary goals of this study were to 1) elucidate behaviors that enhance or diminish health status relative to forest onchocerciasis and related filarial infections, and 2) make culturally sensitive and appropriate recommendations regarding the development of health education materials and the long-term maintenance of the mass distribution of ivermectin in Dja-Lobo.

\section{METHODS}

Study area. The study took place in Dja-Lobo Division, South Province, Cameroon. This area was selected for ivermectin distribution because previous studies conducted by the Institut Francais de Recherche Scientifique pour le Developpement en Cooperation indicated 60-90\% prevalence rates for villages close to the Dja River, the major breeding site for the black fly vector (Simulium damnosum) (Chippeaux P, unpublished data). The ecology of Dja-Lobo is dominated by dense tropical forest with rainfall between 1,500 and 2,000 $\mathrm{mm}$ per year and an average daily high temperature of $25^{\circ} \mathrm{C}$. There are approximately 150,000 people in the division of which the majority (85-95\%) are Boulou, a Bantu-speaking people, while the Baka (pygmies), an 
Oubanguian-speaking people, are an important minority ethnic group in the area ( $0-14 \%$ of village populations). Manioc and plantains are staples for both Boulou and Baka. Fish, game meat, and chickens are the prime sources of protein, while cacao is the most important cash crop. The Baka do some farming but spend considerably more time in the forest hunting and collecting forest products. The Boulou and Baka have complex social and economic relations; the basic element of their economic relationship is that Boulou provide manioc to the Baka in exchange for game meat and other forest products.

The Boulou and Baka have similar demographic features: the average household size is about 5.5 persons and approximately $45 \%$ of the population is less than 15 years of age. Baka seldom practice polygamy, in part, because they often lack the material wealth to pay the bride price, while it is relatively common for the Boulou (16\% of the male heads of household in study village). Baka seldom cultivate cacao or other domestic cash crops, while they are common among the Boulou. Boulou households without cash crops are often headed by a divorced or widowed woman $(53 \%$ of the Boulou households in the study village without cacao were headed by a female). The average income from the previous years' cacao (for households with cacao fields) in the study village was 66,000 Central African Francs (CFA; about \$264).

Other sources of income for Boulou and Baka include the sale of bush meat and other forest products, local government and private sector jobs (logging in particular), and money sent by family members with jobs in Yaounde or Sangmelima.

Baka rarely camp on large rivers, but rather prefer interfluvial sites for hunting and fishing in small streams. Some Boulou men are specialists in fishing, but prefer large fish in or near the Dja or in large tributaries.

The mean prevalence of onchocerciasis in Dja-Lobo is about $75 \%$, but the mean CMFL community microfilarial load (CMFL) is only 2.24 microfilariae $(\mathrm{mf}) / \mathrm{mg}$ of skin. ${ }^{1}$ This compares to West African savanna mean prevalences of $60-88 \%$ and mean CMFLs of $18-81 \mathrm{mf} / \mathrm{mg}$ of skin; even in mosaic forest-savanna environments in West Africa the CMFLs range from 15 to $28 \mathrm{mf} / \mathrm{mg}$ of skin. ${ }^{2}$ The prevalence of Loa loa in South Province is about $30 \%,{ }^{3}$ while the prevalence of one or more soil-transmitted intestinal worms in fifth grade school children in South Province is $98.1 \% .^{4}$ Intestinal worms are a concern of local peoples and may contribute to greater participation in the ivermectin cost recovery progrm if the local people know the new drug kills some intestinal worms.

Techniques. The study consisted of two sequential components, the first primarily qualitative and the second quantitative. The study is unique in that physicians responsible for the supervision and implementation of the project, the second and third authors of this paper, actively participated in the design and implementation of both components of the cultural study, which was directed by the first author, a medical anthropologist.

The qualitative component consisted of a two-day survey of six front-line villages (i.e., villages within $10 \mathrm{~km}$ of the Dja River) near the Dja River and a six-day stay in one of these front-line villages. The two-day survey used focus group interviews to obtain a general overview of Boulou and Baka explanatory models for onchoserciasis. Explanatory models refer to local peoples' understanding and explanations for the disease and includes the study of local peoples' abilities to recognize the disease (e.g., local term for it), the signs and symptoms local people associate with the disease, how local people feel it is transmitted, various therapies people seek to treat the disease, and their perceptions about prognosis. The village study took place in a hyperendemic village of 250 Boulou and 27 Baka approximately $3 \mathrm{~km}$ from the Dja River. The aim was to obtain more in-depth information on explanatory models (see above), and to more systematically evaluate the perceived severity of onchocerciasis. Methods used during the in-depth village component included: 1) household census (age, sex, marital status, kilograms of cacao sold during previous year, and self-reported presence/absence of filariasis, intestinal worms in previous year, and presence/absence of leopard skin and blindness of all household members); 2) a list and description of all known illnesses from eight Boulou and three Baka informants; 3) a ranking technique with 20 Boulou ( 10 males and 10 females) informants to establish the preceived severity of the illnesses desribed by the eight Boulou. The 20 Boulou informants were asked to place 28 illnesses into three groups (severe, sometimes severe, and seldom if ever, severe). Severe was described as potentially leading to death or permanent loss of a bodily function (e.g., ability to walk, have children, or see). After selecting the ranking, each respondent was asked to explain why they were so ranked to better understand concepts of severity. Finally, each respondent was asked to select the three most severe illnesses from the top category, and to rank them by severity. Four (two males and two females) Baka were also asked to place 29 illnesses into two groups (severe versus not severe) and then asked to select and explain the three most serious illnesses from those illnesses placed in the severe category. 4) Open-ended questions were asked about treatment-seeking behavior and activities that take people closer to the Dja River. 5) General participant-observation activities also took place (e.g., visiting fields, observing home treatments and traditional healers application of indigenous herbal treatments for onchocerciasis).

The second component was quantitative and consisted of administering a questionnaire to 212 randomly selected individuals. Drawing upon data generated by the qualitative component, a questionnaire was designed to quantify, specify, and evaluate applicability of the Boulou explanatory model to a broader population in the Dja-Lobo Division. The questionnaire was designed to elucidate information relative to the recognition of onchocerciasis and other filarial diseases, their immediate cause, the mode(s) of acquisition, disease manifestations, treatment options and outcomes, perceptions of severity including willingness to pay for treatment relative to other common diseases, sources of respondents knowledge, and radio ownership. The questionnaire had 32 questions, about half of which required yes/no/do not know responses while the remaining questions required the informant to select one or more items from several alternatives. Basic demographic data were collected for each respondent (male or female head of household) as well as related information about the family unit. 
Due to time and cost restraints it was decided to focus the extensive questionnaire on Boulou and linguistically related Bantu speakers since they represented such an overwhelming majority of the Dja-Lobo population. It was also decided to administer the questionnaire to second (10-20 km from Dja River) and third-line ( $>20 \mathrm{~km}$ from the Dja River) villages rather than front-line ones because more distant villages were far more numerous, and we wanted be certain we had a clear understanding of the perceptions and priorities of the people in villages farther from the river because they would provide the greatest challenge to developing a sustainable ivermectin distribution program. These villages were of concern because there were at least four times as many second- and third-line villages as there were first-line villages, and they often had lower intensity of infection and presumably fewer symptoms. This might decrease treatment seeking and increse the overall cost of delivery (i.e., fewer people participating in cost recovery).

Sangmelima is a city about $125 \mathrm{~km}$ south of the Cameroonian capital of Yaounde. The city is the regional (subdivision) center for four health zones. Two rural villages that had a population of more than 150 were randomly selected from three of the four health zones. One rural village and one section (quartier) of the city were randomly selected from the first health zone that included the city. A total of eight communities participated in the study (seven rural villages and one section of the city).

The questionnaire was prepared first in English and then in French, and experienced health interviewers fluent in French and Boulou were trained to administer the questionnaires. The questionnaires were pretested in the field and modified accordingly. The questionnaire was administered to the 88 male or 124 female heads of household of 212 randomly selected households in the eight communities.

\section{RESULTS}

The results of the qualitative village study and the quantitative questionnaire administered to 212 heads of households in eight communities are summarized below.

Recognition, definition, and transmission. The two-day survey of the qualitative component indicated that most people in the area had some knowledge about onchocerciasis. Minak was the term used for filariasis in general and at least some people believed that the black fly (called nyamendimi) was, in part, responsible for its transmission. This preliminary study also demonstrated the general homogeneity of the ethnolinguistic groups. Boulou, Fang, and several other ethnic groups in the area speak slightly different dialects, but all used the terms minak and nyamendimi. Only the Baka had terms and explanatory models that were different from the Boulou. This was expected since the Boulou speak a Bantu language and are farmers, while Baka speak an Oubanguian language and are foragers.

The quantitative questionnaire indicated that essentially all $(98 \%)$ respondents knew of filariasis (minak), and virtually all $(97 \%)$ believed that the itching was caused by tiny worms biting under the skin. More than $90 \%$ of all respondents identified flies as important vectors of the illness, while fewer people listed mosquitoes (47\%), dirty water (56\%), sorcery $(31 \%)$, and taboo foods $(27 \%)$ as important sources

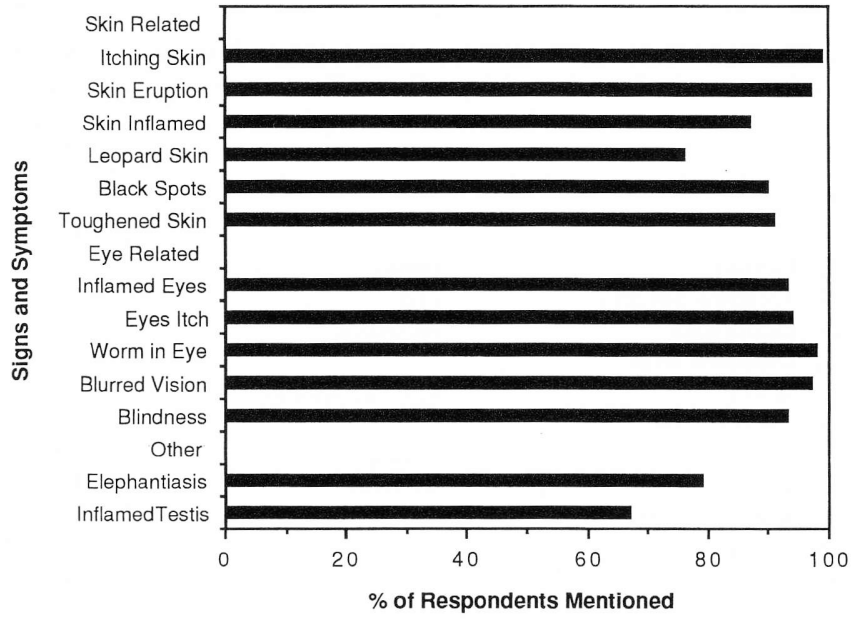

FIGURE 1. Signs and symptoms of minak (general filarasis) in 212 responders in South Province, Cameroon, 1992. Signs and symptoms were divided into three groups: skin-related, eye-related, and other signs and symptoms.

of the illness. Only $6 \%$ thought filariasis could be contracted by touching an infected person, but $81 \%$ indicated maternalfetal transmission was possible and $66 \%$ indicated filariasis could be transmitted sexually.

The informants that identified flies as important vectors for filariasis were asked to specify which species of fly. Most $(96 \%)$ indicated the deer fly (chrysops) could serve as a vector, $55 \%$ identified the black fly (simulium) as a vector, and $33 \%$ thought a tsetse fly could transmit filariasis.

The Baka in the qualitative village study were the first to suggest that leopard skin (ngangambo ) was not linked to filaria (bibili), but rather to illness of the Dja, and sometimes old age. The village study was almost finished when this was mentioned, but it was discussed with several Boulou and they concurred that a primary cause of leopard skin (menve) was illness of the Dja caused by living close to the Dja, getting bitten by black flies, scratching the bites, the bites getting infected and eventually healing. The white spots on the skin were perceived as scarification that takes place as the skin healed.

Signs and consequences. Figure 1 summarizes knowledge of the signs and symptoms of filariasis. Virtually all respondents associated itching (eeyae) and a rash (mebib) with minak (filariasis) and more than $60 \%$ also recognized swelling of the skin and leopard skin (menve) as manifestations of filariasis. With respect to ocular manifestations, nearly all respondents said that swelling and redness of the eye, as well as diminished vision and blindness were consequences of filariasis. Also very important was that virtually all believed that a worm could migrate across the eye with filariasis.

Severity. The ranking exercise with 20 Boulou adults is summarized in Table 1. More than $75 \%$ of respondents placed the following illnesses in the most severe category: madness caused by sorcery, hernia, gonorrhea, sorcery attack for trespassing in others' fields, diarrhea with blood, arthritis, measles, and an object placed in a person by sorcery. More than $75 \%$ of respondents placed the following illnesses in the least severe category: conjunctivitis, scabies, 
TABLE 1

Rank of relative severity of illnesses (from highest to lowest) by 20 informants in front-line village, South Province, Cameroon, 1992

\begin{tabular}{|c|c|c|}
\hline Illness rank* & Boulou term & $\begin{array}{l}\text { Total points } \\
\text { (frequency in } \\
\text { top group) }\end{array}$ \\
\hline 1. Crazy-sorcery & kong & $58(18)$ \\
\hline 2. Hernia & oyong & $57(17)$ \\
\hline 3. Trespass-sorcery & eya & $55(16)$ \\
\hline 4. Gonorrhea & menyock & $55(16)$ \\
\hline 5. Diarrhea with & & \\
\hline $\begin{array}{l}\text { blood } \\
\text { 6. Rheumatism and }\end{array}$ & ntui na metchi & $54(15)$ \\
\hline arthritis & akang & $54(15)$ \\
\hline 7. Measles & sabiasa & $53(15)$ \\
\hline 8. Spear-sorcery & nsong & $53(15)$ \\
\hline 9. Prolapsed rectum & $t s a^{\prime} a$ & $51(11)$ \\
\hline 10. Severe cough $(\mathrm{TB}) \dagger$ & ekoe & $50(12)$ \\
\hline 11. Blindness & ndim & $47(13)$ \\
\hline 12. Abscess & etchel & $47(9)$ \\
\hline 13. Malaria & tit & $45(9)$ \\
\hline 14. Hepatitis & zom & $45(10)$ \\
\hline 15. Filariasis & minak & $44(9)$ \\
\hline 16. Diarrhea & ntui & $44(8)$ \\
\hline 17. Intestinal worms & misong & $43(8)$ \\
\hline 18. Listlessness/fever & koe ebem & $43(8)$ \\
\hline 19. Spirit eats & awa mbim & $42(8)$ \\
\hline 20. Uterine pains & nsong nyo & $39(5)$ \\
\hline 21. Yaws & mebata & $39(4)$ \\
\hline 22. Dental caries & mesong mintae & $38(5)$ \\
\hline 23. Cold/flu & mboman & $37(5)$ \\
\hline 24. Elephantiasis & mendoung & $36(6)$ \\
\hline 25. Goiter & ovuk & $35(5)$ \\
\hline 26. Tropical ulcer & aveng & $30(5)$ \\
\hline 27. Scabies & mintchang & $25(0)$ \\
\hline 28. Conjunctivitis & bibolo & $23(0)$ \\
\hline
\end{tabular}

* Some of the illnesses are primarily supernatural and may need further explanation. 1 . Crazy: a sorcerer sends a poison dart into his/her victim that induces madness and eventual death; 3. Trespass: an individual trespasses and steals from another's fields and is attacked pear or pointed object into a victim that results in illness and death unless it is invisible 19. Spirit eats: spirits of the dead take food from family and death unless it is removed the victim gets thinner and weaker. $+\mathrm{TB}=$ tuberculosis

and tropical ulcer. Filariasis, malaria, worms, and blindness were in the middle range overall, but were not infrequently included in the most severe category (i.e., they can sometimes be severe).

None of the Baka placed bibili (= minak in Boulou, filariasis) or sassa (itching all over) into the serious illness category. Sassa was seen as the primary symptom of filariasis (bibili) and is believed to be transmitted by wosili (chrysops). Baka did not think filariasis caused blindness, nor that it is linked to eye-worms ( $L$. loa).

The quantitative questionnaire asked respondents to rank the following illnesses in degree of severity $(1=$ highest to 5 = lowest): malaria, blindness, filariasis, intestinal worms, and diarrhea. Table 2 summarizes the results and is consistent with the results from the more detailed method in Table 1. Blindness was considered by far the most severe in this group.

Susceptibility. Sixty-two percent of the questionnaire respondents indicated adults were at more risk of filariasis than were children. Children were reported to have a greater susceptibility to intestinal worms. This was consistent with the qualitative village study in which heads of households reported that $80 \%$ of the Boulou and Baka adults had had filariasis in the previous year, but only $5 \%$ of the Boulou
TABLE 2

Ranking of five illnesses (from highest $=\# 1$ to lowest $=\# 5$ ) by 212 individuals, South Province, Cameroon, 1992

\begin{tabular}{lcc}
\hline \multicolumn{1}{c}{ Illness } & Mean score & Frequency ranked \#1 \\
\hline Blindness (ndim) & 2.0 & 134 \\
Filaria (minak) & 2.6 & 29 \\
Malaria (tit) & 2.8 & 40 \\
Diarrhea (ntui) & 3.8 & 4 \\
Intestinal worms & & \\
$\quad$ (misong) & 3.9 & 3 \\
\hline
\end{tabular}

children and $0 \%$ of the Baka children had had filariasis in that time period. The reverse pattern was found with intestinal worms: $71 \%$ of the Boulou adults and $60 \%$ of the Baka adults had had intestinal worms in the previous year, while more than $90 \%$ of the Boulou children and all of the Baka children reportedly had had intestinal worms in that same period.

Treatment and cost. More than $90 \%$ of questionnaire respondents reported that they had had filariasis at least once, and of these $69 \%$ reportedly had sought treatment. Of those who sought treatment, $41 \%$ had sought treatment during the previous 12 months. Most respondents had tried both traditional and Western treatments. Fifty-four percent had tried some form of traditional relief while $50 \%$ had tried Notezine $^{(\mathbb{N A})}$ (diethylcarbamazine [DEC]; Lederle, Wayne, NJ), 49\% had tried Phenergan (1W (promethazine hydrochloride; Wyeth-Ayerst, Philadelphia, PA), and 38\% had tried M.G. Lumiere (the last two relieve itching).

Respondents did not seem to be satisfied with either traditional or existing Western medications. Eighty-one percent said traditional treatments (e.g., several herbal creams and liquids placed on itching areas) did not cure filariasis and $80 \%$ said Notezine did not cure the disease. Sixty-nine percent said traditional treatment did not stop the itching and $44 \%$ said DEC was not effective. While $50 \%$ of the Boulou who had filariasis said they took DEC as a treatment, these same individuals reported several side effects and fears about taking the drug. Fatigue was the most commonly mentioned side effect (60\%), but increased itching (33\%), body pain $(32 \%)$, exacerbation of other illnesses (especially malaria) $(38 \%)$, and fever $(35 \%)$ were also noted side effects. Respondents were also asked if there were any other potential side effects, and more than $60 \%$ of the 40 respondents that spontaneously gave another side effect indicated that it was possible to die after taking this drug!

Baka in the qualitative village study said they did not seek specialized treatment for filariasis (bibili). Baka indicated that the itching from filariasis disappeared eventually even without traditional treatment; none of the Baka mentioned going to the health center $2 \mathrm{~km}$ away for treatment even though $80 \%$ of the adults reported having had filariasis in the previous year.

There was considerable variability in how much respondents to the questionnaire paid to obtain treatment the last time they had had filariasis. Most (36\%) said they paid more than 1,000 CFA (about \$4), but 28\% said they paid nothing for their traditional or home treatment. Most respondents $(66 \%)$ received their treatment at a private health center and only $28 \%$ received treatment at a public health center. Many 
people were able to obtain treatment in the village or within a $5-\mathrm{km}$ walking distance $(65 \%)$, but more than $22 \%$ traveled more than $30 \mathrm{~km}$ to obtain treatment.

Respondents were also asked how much they paid for the treatment of other common illnesses, such as malaria, diarrhea, and intestinal worms. More than $60 \%$ of those interviewed said they paid more than $600 \mathrm{CFA}(\$ 2)$ to obtain treatment for these illnesses. When asked how much they would be willing to pay for treatment of filariasis most (51\%) of informants indicated something between 100 and 300 CFA would be a reasonable amount. Ninety-three percent said they would be willing to pay this amount on a yearly basis.

Health communication. Most respondents indicated that they had learned about filariasis from a mixture of information from family/relatives $(50 \%)$ and personal experience $(96 \%)$. Informants often commented that when they got filariasis, they began to experience itching and that it felt like there were worms under their skin.

Sixty-eight percent of the heads of households reported that they had a radio in the house, but only half of these respondents indicated that the radio was functioning. Forty percent reportedly talked with a health worker about filariasis and $43 \%$ said they had seen posters or other health education materials about this disease.

Independent variables. There were few significant effects due to age, sex, or urban versus rural living. Men and women, young and old, and rural and urban peoples all had similar knowledge and perceptions regarding filariasis. Women were more likely than men to obtain a diagnosis for filariasis from a traditional healer and reported fewer side effects from DEC than did men. Men were more likely than women to report owning a working radio.

\section{DISCUSSION}

The data provided valuable insights, leading to a series of conclusions and related recommendations.

A health education program could build upon existing indigenous knowledge as essentially all Boulou and Baka defined general filariasis as small worms under the skin, identified flies as important transmitters of the illness, and indicated that blindness and other skin and ocular problems were a consequence of the illness. However, serious problems could emerge if such a program relied too heavily on indigenous conceptions because Boulou minak and Baka bibili refer to all filarial diseases. Worms migrating across the eye and Calabar swellings (transient painful red swelling, usually on limbs and near joints) described by the people as linked to filariasis, are clearly the result of L. loa and not onchocerciasis. The number of people that believed DEC could potentially cause death also indicates filariasis to them means L. loa rather than onchocerciasis.

Consequently, the following health education message was recommended: ivermectin treatment annually will prevent the illness of the Dja, which is characterized by leopard skin, and is found mainly in villages near the Dja with many black flies. Targeting minak (general filariasis rather than illness of the Dja) for ivermectin therapy could potentially discredit the ivermectin distribution program because ivermectin would not cure or eliminate clinical manifestations of L. loa infection, namely worms migrating across the eye, and Calabar swelling. Furthermore, epidemiologic data from Dja-Lobo indicated that while most of the area is classified as hyperendemic on the basis of skin snip positivity, only front-line communities have high infection intensity and a history of high black fly densities. Although many of the second and third-line communities would be classified as hyperendemic on the basis of skin snip positivity, the intensity of infection is very low, as are most manifestations of disease. Villagers in many of the second- and third-line communities stated that they had no black flies. At the same time villagers clearly stated that black flies were most common near the Dja, where illness of the Dja was common. In short, the people living in hyperendemic villages away from the Dja suffer relatively little symptomatology from onchocerciasis, and do not recognize onchocerciasis as such as a problem. They recognize filariasis as a complex of symptoms including $L$. loa as well as onchocerciasis.

Several ivermectin distribution programs use health messages that promote ivermectin as a miracle drug for filariasis. This may be a reasonable strategy in the short-run, especially in savanna areas without $L$. loa, but in tropical forest areas indigenous terms may not distinguish $L$. loa from onchocerciasis and such claims may, therefore, undermine the longterm sustainability and credibility of the program.

Health messages that indicate ivermectin prevents blindness and reduces skin problems would also be useful. Filariasis has both skin and ocular manifestations (Figure 1). This is unlike the Guatemalan onchocerciasis cultural-behavioral study ${ }^{5}$ in which eye-related illnesses/symptoms were much more commonly reported than were skin-related illnesses/symptoms. Blindness consistently ranked high in severity studies and should be included in health education, but skin manifestations may also be important because a recent Nigerian study ${ }^{6}$ in the forest-savanna mosaic points out that women with onchodermatitis have a more difficult time finding a spouse. In Nigeria, the bite of the black fly was linked to nodules, but not leopard skin. Nodules were also commonly linked to onchocerciasis in Guatemala, but this was due primarily to the government nodulectomy program that took place before the cultural-behavioral study.

Filariasis is perceived as a greater problem for adults than it is for children even though parasitologic studies in DjaLobo have demonstrated that there are no significant differences in prevalences in children and adults. The following health education messages were recommended to encourage parents to purchase annual treatments for their children: 1) ivermectin kills many kinds of worms (misong) including those in the intestine as well as those under the skin, and 2) children need to be treated each year with ivermectin (even though they do not have evidence of disease) to prevent damage from illnesses such as minak and illness of the Dja and also to kill intestinal worms. Virtually all respondents in the quantitative survey knew that itching was caused by tiny worms under the skin and $98.1 \%$ of the fifth grade school children had at least one type of intestinal worm. ${ }^{4}$ During the previous year, more than $90 \%$ of the households in the questionnaire purchased some Western worm medication for one or more family members. Since misong refers to all worms irrespective of size and location, health educators could take advantage of this linguistic linkage by con- 
veying the information that ivermectin kills intestinal worms as well as the tiny worms under the skin. Health educators in Cameroon now use these messages, but an evaluation of their utility has not taken place.

Onchocerciasis may not be perceived as a very serious illness. Filariasis ranked 15 th of 28 possible illnesses in the qualitative village study. Several supernatural (various forms of sorcery, madness) and natural illnesses were ranked above filariasis. Filariasis, malaria, and blindness were ranked in a middle-range of severity. The few Baka in the village study all stated they had never sought treatment when they were ill with filariasis. The results indicated that $32 \%$ of the Boulou never sought treatment for filariasis. The questionnaire asked individuals to rank only five illnesses; blindness was clearly perceived as the most severe, followed by filariasis and malaria. The study questionnaire used the term minak, when in fact menve (leopard skin) or illness of the Dja may have, in retrospect, been more specific to onchocerciasis.

The question of severity is important for developing a sustainable program. It is likely that the initial distribution efforts will be successful in Dja-Lobo because of extensive TV and radio coverage of the new drug and additional training of local health authorities. Long-term sustainability is another question. Filariasis ranked in the middle range of severity and leopard skin, which is more closely tied to black flies, was certainly ranked even lower. Ivermectin will not remedy many of the L. loa symptoms associated with minak, and a recent placebo-controlled study in Sierra Leone ${ }^{7}$ from a forest onchocerciasis area similar in prevalence (greater than $80 \%$ for adults) and intensity (1-10 mf) to our target population indicates that ivermectin does not reduce the burden of itching. Whitworth and others ${ }^{8}$ concluded that "the lack of obvious benefit to a target population after the first dose of ivermectin may reduce compliance with subsequent doses."

Most Boulou are willing to pay 100-300 CFA (\$1) for an annual ivermectin treatment. Many people pay this amount several times a year for illnesses that are considered less severe (e.g., intestinal worms and diarrhea). The problem is that somewhat older (30 years of age and older) single women (due to divorce or death of spouse) and Baka, which represent $10-25 \%$ of village households, generally do not have cash crops and may have other priorities for the limited amount of cash they do have.

Finally, it should be noted that this study is unusual in that physicians responsible for the supervision and implementation of the project actively participated in the design and implementation of the cultural and behavioral studies. Rosenfield's recent review ${ }^{9}$ of the cultural and behavioral factors of disease advocates this type of active and interactive transdisciplinary research. She suggests moving beyond conventional multidisciplinary approaches in which researchers from different disciplines do their work independently and then report their findings back to the group. Seldom do multidisciplinary researchers understand each other's methods or contributions. Rosenfield advocates transdisciplinary research, like that reported here, in which health care providers and biomedical researchers participate in aspects of the social science research and vice versa. While this study certainly has limitations, it does demonstrate what an interactive and truly collaborative transdisciplinary research team can do in a relatively short amount of time.

Acknowledgments: We acknowledge the assistance and support of Rene Owona, Jean Bangob, and Richard Green.

Financial support: This research was supported by the International Eye Foundation and USAID-Cameroon.

Authors' addresses: Barry S. Hewlett, Department of Anthropology, Washington State University, Pullman, WA 99164. Basile Kollo, Cameroon Ministy of Health, BP 106, Doula, Cameroon. Barnett L. Cline, Center for International Community-Based Studies, Department of Tropical Medicine, Tulane University School of Public Health and Tropical Medicine, New Orleans, LA 70112.

\section{REFERENCES}

1. Kollo B, Mather FJ, Cline BL, 1995. Evaluation of alternative methods of rapid assessment of endemicity of Onchocerca volvulus in communities in southern Cameroon. Am J Trop Med Hyg 53: 243-247.

2. Zimmerman PA, Dadzie KY, De Sole G, Remme J, Soumbey Alley E, Unnasch TR, 1992. Onchocerca volvulus DNA probe classification correlates with epidemiologic patterns of blindness. J Infect Dis 165: 964-968.

3. Gordon RM, Kershaw WE, Crewe W, Oldroyd H, 1950. The problem of loiasis in West Africa with special reference to recent investigations at Kumba in the British Cameroons and at Sapele in southern Nigeria. Trans $R$ Soc Trop Med Hyg 44: 11-47.

4. Ratard RC, Kouemeni LE, Ekani Bessala MM, Ndamkou CN, Sama MT, Cline BL, 1991. Ascariasis and trichuriasis in Cameroon. Trans R Soc Trop Med Hyg 85: 84-88.

5. Richards R, Klein RE, Gonzales-Peralta C, Zea Flores, R, Zea Flores G, Ramirez JC, 1991. Knowledge, attitudes and perceptions (KAP) of onchocerciasis: a survey among residents in an endemic area in Guatemala targeted for mass chemotherapy with ivermectin. Soc Sci Med 32: 1275-1281.

6. Amazigo UO, 1994. Detrimental effects of onchocerciasis on the marriage age and breast-feeding. Trop Geogr Med 46: 322-325.

7. Whitworth JAG, Duty AJF, Maude GH, Morgan D, Downham MD, Taylor DW, 1992. Ivermectin does not reduce the burden of itching in an onchocerciasis endemic community. Trans $R$ Soc Trop Med Hyg 86: 281-283.

8. Whitworth JAG, Morgan D, Maude GH, Downham MD, Taylor DW, 1991. A community trial of ivermectin for onchocerciasis in Sierra Leone: clinical and parasitological responses to the initial dose. Trans $R$ Soc Trop Med Hyg 85: 92-96.

9. Rosenfield PL, 1992. Social determinants of tropical disease. Warren KS, Mahmoud AAF, eds. Tropical and Geographic Medicine. New York: McGraw-Hill, 197-205. 